\title{
Potencial da terapia por fotobiomodulação no tratamento da atrofia do músculo esquelético
}

\author{
Potential of photobiomodulation therapy in the treatment of skeletal muscle atrophy \\ Potencial de la terapia de fotobiomodulación en el tratamiento de la atrofia del músculo esquelético
}

Recebido: 22/12/2020 | Revisado: 29/12/2020 | Aceito: 30/12/2020 | Publicado: 04/01/2021

\author{
Silma Rodrigues Gonçalves \\ ORCID https://orcid.org/0000-0001-8645-262X \\ Universidade Brasil, Brasil \\ E-mail:_silmaanato@gmail.com \\ Carla Roberta Tim \\ ORCID: https://orcid.org/0000-0002-4745-9375 \\ Universidade Brasil, Brasil \\ E-mail: carla.tim@universidadebrasil.edu.br \\ Cintia Cristina Santi Martignago \\ ORCID: https://orcid.org/0000-0003-3980-6354 \\ Indústria Brasileira de Equipamentos Eletromédicos, Brasil \\ E-mail: csantimartignago@yahoo.com.br \\ Marcelo Cavenaghi Pereira Silva \\ ORCID: https://orcid.org/0000-0002-3270-5086 \\ Universidade Federal de São Paulo, Brasil \\ E-mail: marcaven@gmail.com \\ Carlos Alberto Anaruma \\ ORCID: https://orcid.org/0000-0001-8201-5019 \\ Universidade Estadual Paulista, Brasil \\ E-mail: anaruma@rc.unesp.br \\ Lívia Assis Garcia \\ ORCID: https://orcid.org/0000-0002-8343-3375 \\ Universidade Brasil, Brasil \\ E-mail: livia.assis@universidadebrasil.edu.br
}

\begin{abstract}
Resumo
A atrofia do tecido muscular estriado esquelético é um processo complexo causado por um desequilíbrio entre a degradação e síntese de proteínas miofibrilares, levando à redução da força muscular e, consequentemente, influencia o comportamento emocional, a saúde mental e a qualidade de vida dos indivíduos. Desta forma, a atenuação da atrofia e estímulo para formação de um novo tecido muscular é um desafio para reabilitação. Esforços consideráveis têm sido dedicados ao estabelecimento de novos tratamentos, entretanto, ainda faltam dados experimentais e clínicos confiáveis para sua aplicação clínica. Dentre as recursos terapêuticos disponíveis, a terapia por fotobiomodulação (PBMT, do inglês photobiomodulation therapy) apresenta um grande potencial, visto que é frequentemente utilizada como estratégia terapêutica promissoras para a reabilitação do tecido muscular estriado esquelético. Dentro deste contexto, o objetivo deste estudo é proporcionar, através de uma revisão narrativa, uma compreensão das evidências atuais disponíveis sobre a importância da PBMT no tratamento de atrofia do músculo esquelético. As buscas foram realizadas nas bases de dados bibliográficas do PubMed/MEDLINE, Biblioteca virtual da saúde (BVS), Web of Science e SciELO. As evidências encontradas neste estudo direcionam que a PBMT pode ser proposta como uma intervenção terapêutica eficaz no tratamento da atrofia muscular, devido ao potencial de estimular fatores regulatórios miogênicos que promovem a ativação e proliferação de células satélites e consequente aumento da formação de fibras musculares, assim como atenuar e apoptose de celular as vias de proteólise da fibra muscular.
\end{abstract}

Palavras-chave: Fototerapia; Lasers; Atrofia muscular; Reabilitação.

\begin{abstract}
Atrophy of striated skeletal muscle tissue is a complex process caused by an imbalance between the degradation and synthesis of myofibrillar proteins, leading to a reduction in muscle strength and, consequently, influencing the emotional behavior, mental health and quality of life of individuals. Thus, the attenuation of the atrophy and stimulus for the formation of new muscle tissue is a challenge for rehabilitation. Considerable efforts have been devoted to the establishment of new treatments, however, reliable experimental and clinical data are still lacking for its clinical application. Among the therapeutic resources available, photobiomodulation therapy (PBMT, from the English photobiomodulation therapy) has great potential, as it is often used as a promising therapeutic strategy for the rehabilitation of skeletal muscle tissue. Within this context, the aim of this study is to provide, through a narrative review, an understanding of the current available evidence on the importance of PBMT in the treatment of skeletal muscle atrophy. The searches were performed in the bibliographic databases of PubMed / MEDLINE, Virtual Health
\end{abstract}


Library (VHL), Web of Science and SciELO. The evidence found in this study points out that PBMT can be proposed as an effective therapeutic intervention in the treatment of muscle atrophy, due to the potential to stimulate myogenic regulatory factors that promote the activation and proliferation of satellite cells and the consequent increase in the formation of muscle fibers, as well as how to attenuate and cellular apoptosis the proteolysis pathways of muscle fiber.

Keywords: Phototherapy; Lasers; Muscle atrophy; Rehabilitation.

\section{Resumen}

La atrofia del tejido del músculo esquelético estriado es un proceso complejo provocado por un desequilibrio entre la degradación y síntesis de las proteínas miofibrilares, que conduce a una reducción de la fuerza muscular y, en consecuencia, influye en el comportamiento emocional, la salud mental y la calidad de vida de los individuos. Por tanto, la atenuación de la atrofia y el estímulo para la formación de nuevo tejido muscular es un reto para la rehabilitación. Se han dedicado considerables esfuerzos al establecimiento de nuevos tratamientos, sin embargo, aún faltan datos experimentales y clínicos confiables para su aplicación clínica. Entre los recursos terapéuticos disponibles, la terapia de fotobiomodulación (PBMT, del inglés terapia de fotobiomodulación) tiene un gran potencial, ya que a menudo se utiliza como una estrategia terapéutica prometedora para la rehabilitación del tejido del músculo esquelético. En este contexto, el objetivo de este estudio es proporcionar, a través de una revisión narrativa, una comprensión de la evidencia disponible actualmente sobre la importancia de PBMT en el tratamiento de la atrofia del músculo esquelético. Las búsquedas se realizaron en las bases de datos bibliográficas de PubMed / MEDLINE, Virtual Health Library (VHL), Web of Science y SciELO. La evidencia encontrada en este estudio indica que el PBMT puede proponerse como una intervención terapéutica eficaz en el tratamiento de la atrofia muscular, debido al potencial de estimular factores reguladores miogénicos que promueven la activación y proliferación de células satélite y el consecuente aumento en la formación de fibras musculares, así como cómo atenuar y apoptosis celular las vías de proteólisis de la fibra muscular.

Palabras clave: Fototerapia; Láseres; Atrofia muscular; Rehabilitación.

\section{Introdução}

A perda de massa muscular ou atrofia muscular pode estar associada à idade ou a diversas condições clínicas como o câncer, diabetes mellitus, doenças cardíaca e pulmonares, síndrome de imunodeficiência adquirida (HIV), sepse, denervação, distrofias musculares, imobilização prolongadas assim como, mais recentemente evidenciado na doença causada pelo novo coronavírus (COVID-19) (Bodine \& Baehr, 2014; Lee \& Jun, 2019; Rosa-Caldwell et al., 2019; Morley et al., 2020).

Estima-se que esse tipo de alteração musculoesquelética aumentou $46 \%$ nos últimos anos devido ao envelhecimento populacional e internações de longo prazo, afetando diretamente a qualidade de vida e com aumento da mortalidade entre 20 e 40\% (March et al., 2014; Beaudart et al., 2017; Gruet et al., 2017; Lee et al., 2019).

Neste sentido, pesquisas que tenham como objetivo desenvolver intervenções terapêuticas que possam atenuar o processo de atrofia, estimular o processo regenerativo ou ainda favorecer a recuperação estrutural e funcional do tecido muscular são de extrema importância.

Atualmente, a terapia por fotobiomodulação (PBMT) através de lasers (amplificação de luz por emissão estimulada de luz) e LEDs (diodos emissores de luz) tem sido considerada uma técnica segura e eficiente para o tratamento de uma variedade de doenças e lesões musculoesquelética pois apresenta propriedade analgésica, moduladora do processo inflamatório e reparador (Kou et al., 2019). Sabe-se que a luz monocromática penetra nos tecidos e são absorvidas por fotorreceptores celulares específicos, denominados cromóforos promovendo diversas modificações celulares e moleculares (Chung et al., 2012; de Freitas \& Hamblin, 2016), como incremento do potencial de membrana mitocondrial e síntese de adenosina trifosfato (ATP), prevenção e reparação de danos musculares, estimulação de defesas contra o estresse oxidativo e o aumento do desempenho da fibras musculares (Assis et al., 2013; Ferraresi et al., 2012; Ferraresi et al., 2015).

Considerando que a necessidade de fornecer subsídios para a implementação de planos de intervenções mais específicos e direcionados para a reabilitação de pacientes com atrofia muscular, o objetivo deste estudo é proporcionar uma compreensão das evidências atuais disponíveis a respeito da importância PBMT no tratamento da atrofia do músculo esquelético. 


\section{Referencial Teórico}

\section{Músculo esquelético e atrofia muscular}

O músculo esquelético é o principal constituinte do sistema músculo-esquelético, um dos maiores sistemas orgânicos do corpo, respondendo por 40-50\% do peso corporal total (Brook et al., 2016). Esse, contribui para uma variedade de funções corporais, sendo que sua principal função é converter energia químicas em energia mecânica para gerar força e potência, manter a postura e produzir movimentos. De um perspectiva metabólica, contribui com o metabolismo energético basal, sendo fonte de glicose e substratos importantes, tais como os aminoácidos e carboidratos, que serão disponibilizado ao organismo diante de situações de estresse energético (Frontera \& Ochala, 2015; Wolfe, 2006).

São derivados derivado de células precursoras do mesoderma paraxial embrionário a partir de somitos originados nas laterais do tubo neural durante a embriogênese dos vertebrados (Chal et al., 2018). Os somitos desenvolvem-se de forma rostro-ventral e as células somíticas iniciam a expressão de genes progenitores que irão ativar programas de diferenciação miogênica (Chal \& Pourquié, 2017; Hubaud \& Pourquié, 2014) formando os miótomo, que subsequentemente se fundem formando os miotubos e as fibras musculares (Chal et al., 2018). Além disso, é conhecido que o crescimento muscular pósnatal ocorre devido a presença de células satélites (CS), célula precursora miogênica localizadas na periferia das fibras musculares entre a lâmina basal e o sarcolema, as quais se diferenciam, proliferam-se e se fundem com as fibras pré-existentes (Frontera \& Ochala, 2015; Musarò \& Carosio, 2017).

O sistema muscular é formado por células denominadas fibras musculares ou miócitos, de características contráteis, cilíndricas e alongadas, estriadas com núcleos periféricos e multinucleada. O citoplasma ou sarcoplasma é constituído principalmente por miofibrilas contráteis que se estendem por toda fibra e o arranjo das mesmas são responsáveis pelas estriações transversais e formação das unidades funcionais do músculo denominadas de sarcômeros. Estes são compostos principalmente por duas proteínas: os filamentos grossos de miosina e os filamentos finos de actina. É através da interação desses dois filamentos de proteína ocorre o fenômeno de contração muscular (actina deslizando sobre a miosina) (Ono, 2010).

Sabe-se que no tecido muscular há três camadas de tecido conjuntivo (também chamado de tecido conectivo) que se unem nas extremidades dos músculos para formar os tendões e/ou fixam em outras estruturas. Estes tecidos são organizados da seguinte forma: epimísio - camada externa, que separa o músculo dos tecidos e órgãos adjacentes, aderido a fáscia muscular (profunda) que envolve todo músculo esquelético e contém muitos vasos saguíneos e nervos; perimísio - envolve os feixes de fibras musculares, dividindo o músculo em compartimentos internos, fascículos (feixe) musculares; endomísio - camada mais interna que envolve cada célula (miofibra) muscular (Monte Alegre et al., 2012).

As fibras musculares deste tecido diferem em sua composição molecular e propriedades estruturais e funcionais (Schiaffino \& Reggiani, 2011). Estas, podem ser classificadas com base na velocidade de contração, capacidade metabólica e expressão da cadeia pesada de miosina (MyHC) (Spangenburg \& Booth, 2003). Classicamente, as fibras são descritas em fibras vermelhas (tipo I) ou brancas (tipo II). As fibras vermelhas, ricas em mitocôndrias e mioglobinas, com metabolismo oxidativo e atividade tônica e as fibras brancas, pobre em mitocôndrias e mioglobinas, com metabolismo glicolítico e atividade fásica (Schiaffino, 2011).

É possível encontrar na literatura diversos subtipos de fibras musculares, diferindo nas diferentes espécies, entre os indivíduos e ainda nas diferentes regiões do corpo. Neste sentido, quatro principais tipos de fibras distribuídos em músculos do corpo de mamíferos foram descritas: Tipo I, IIa, IIx/d e IIb (Jagoe \& Goldberg, 2001; Schiaffino \& Reggiani, 2011). As fibras do tipo IIx têm o tempo de contração e relaxamento semelhantes às das unidades IIa e IIb, e sua resistência à fadiga é intermediária entre as IIa e IIb (Schiaffino \& Reggiani, 2011).

É um tecido que apresenta alta capacidade de se adaptar dependendo de suas demandas funcionais. Vários fatores que podem intervir na arquitetura e/ou na composição fibrilar da musculatura estriada esquelética, tais como, como exercícios 
(Fink et al., 2018; Fink et al., 2018), suplementos nutricionais e compostos químicos (Aoki et.al., 2006; Baptista et al., 2017), doenças (Cohen et al., 2015; Joglekar et al., 2015), entre outros. Estes fatores geralmente podem induzir adaptações do tipo hipertrofia, hipotrofia e até a atrofia muscular.

A hipertrofia é caracterizada pelo aumento no volume do tecido, células, contendo quantidade acrescentada de proteínas estruturais e de organelas, que resulta em aumento do tamanho do órgão; a hipotrofia é a redução no volume do tecido ou de células; e a atrofia é a redução funcional de um órgão, com redução do volume ou do número de células, pela perda de substância celular, embora as células atrofiadas tenha sua função reduzida, elas não estão mortas (Boonyarom \& Inui, 2006; Hindi et al., 2013).

Já, a atrofia do músculo esquelético é caracterizada por um desequilíbrio entre a síntese e a degradação proteica (proteólise), a favor da degradação, sendo considerada a principal causa de perda da massa muscular decorrente de redução da área das fibras musculares e/ou redução da quantidade de fibras musculares (Yao et al., 2015; Gao et al., 2018). Diversas situações podem estar associadas a um estado catabólico, incluindo as adaptações devido condições fisiológicas do organismo como envelhecimento, jejum prolongado, inatividade, imobilismo, bem como condições patológicas tais como desnervação, septicemia, diabetes, caquexia, microgravidade, AIDS, alterações renais, sepse, doença sistêmica, lesões térmicas e medulares, doenças degenerativas (Boonyarom \& Inui, 2006; Bodine \& Baehr, 2014; Lee \& Jun, 2019).

Nestes processos várias alterações ocorrem no tecido muscular como o encolhimento das miofilbras devido a redução das proteínas contráteis, organelas e citoplasma, redução da área de seção transversa (AST) da fibra muscular, transição das fibras de contração lenta (tipo I) para as fibras de contração rápida (tipo II) e aumento da resistência à insulina e degradação de proteínas miofibrilares (Zhang et al., 2007; Schiaffino et al. 2013; Bodine \& Baehr, 2014; Wang et al., 2017).

Estudos mostram que o imobilismo/desuso (umas das razões frequentes encontradas clinicamente) levam a uma atrofia muscular de aproximadamente 0,5\% da massa muscular total por dia (Wall \& Van Loon, 2013). da Silva et al., (2006), demostraram que após quatro dias de imobilização do tornozelo de ratos ocorrem alterações dos sarcômeros das fibras musculares do tipo I, e que no sétimo dia foi observado uma diminuição do diâmetro das fibras musculares, aumento da densidade do tecido conjuntivo intramuscular e diminuição do glicogênio em diversos músculos da perna (Järvinen et al., 2002; da Silva et al., 2006).

O mecanismo de proteólise envolve a ativação de diversas vias celulares, tais como, as vias lisossômica, dependente de cálcio e o sistema ubiquitina-proteossoma (UPS) (Scicchitano et al., 2015; Baehr et al., 2017). Dentre todas as vias envolvidas na atrofia muscular, a sistema UPS é a principal via reguladora de degradação de proteínas intracelulares no músculo esquelético (Jagoe \& Goldberg, 2001; Bodine \& Baehr, 2014; Scicchitano et al., 2015; Baehr et al., 2017). Pesquisas demonstram que a atrofia muscular que ocorre na imobilização está associada à ativação das vias do sistema UPS (Aoki et al., 2006; Wall et al., 2014),

O UPS contribui para a atrofia muscular por meio de uma redução na síntese de proteína e/ou aumento da degradação da mesma. A ação proteolítica do UPS é dependente de ATP e envolve três enzimas distintas na quebra de proteínas miofibrilares: a ligase E1 (enzima ativadora de ubiquitina), ligase E2 (enzima de conjunção da ubiquitina) e a ligase E3 (enzima ubiquitina-ligase) (Gao et al., 2018).

Sabe-se que durante o processos de atrofia muscular a ubiquitina ligase 3 é considerada a enzima de regulação primária da proteólise muscular.Nessas condições há um aumento das enzimas músculo-específicas conhecidas como: muscle RING finger 1 (MuRF1) e muscle atrophy F-box (MAFbx, também conhecida como atrogina-1) (Brooks \& Myburgh, 2014; Gao et al., 2018).

MuRF1 e atrogina (MAFbx) desempenham um papel essencial no início do processo da atrofia, visto que é observado um aumento da expressão de ambos em vários modelos de atrofia muscular (Wall et al., 2014; Baehr et al., 2017). Estudos 
mostram que a atrogina-1 atua na diminuição da síntese proteica visto que esta inibinde a formação dos miotubos após bloqueio da MyoD. MuRF1 interage preferencialmente com proteínas estruturais e promove a degradação de proteínas do sarcômero incluindo titina, miosina, nebulina e troponina; também atua no controle da degradação de outras proteínas musculares (Gao et al., 2018).

Baptista et al. (2017) demostraram que três dias após a imobilização do membro pélvico, os níveis de mRNA de MuRF1, foram elevados em 3,5 vezes no músculo sóleo em ratos. Resultados semelhantes foram encontrados em humanos, após a imobilização do joelho, a expressão de mRNA do MuRF1 aumentou após 5 dias no músculo vasto lateral, e a expressão de mRNA da atrogina-1 aumentou após 5 e 14 dias de desuso (Wall et al., 2014).

\section{Regeneração muscular após atrofia}

Para que haja o restabelecimento da fibra muscular atrofiada é necessário que o ocorra a miogêneses, ou seja, a formação de uma fibra muscular (Musarò \& Carosio, 2017). Neste processo é necessário a participação de uma pequena população de células mononucleadas ou estaminais, as CS, que fazem com que o músculo esquelético seja dinâmico e capaz de responder aos estímulos fisiológicos ou patológicos. Estas células são frequentemente encontradas estado de quiescente e serão ativadas pela ação coordenada de fatores regulatório miogênicos (MRFs), fazendo com que estas voltem ao ciclo celular em resposta às necessidades de renovação mionuclear e hipertrofia da miofibra, restabelendo a arquitetura das fibras musculares atrofiadas (Frontera \& Ochala, 2015).

Os MRFs são compostos por: Myf5, MRF4 (Myf6), MyoD e miogenina (MyoG), os quais são fatores de transcrição nuclear controlados por diversos genes músculo-específicos. Estas proteínas pertencem a uma família selecionada de fatores de transcrição básico hélice-alça-hélice (basic helix-loop-helix - bHLH) (Hernández-Hernández et al., 2017; Zammit, 2017). Devido seus diferentes papéis essenciais desempenhados durante a embriogênese e miogênses pós-natal, os MRFs foram divididas em dois grupos funcionais. Os MRFs primários, MyoD e Myf-5 são reguladores essenciais da determinação da linhagem muscular estriada esquelética (Kablar et al., 1999; Abreu et.al., 2017; Hernández-Hernández et al., 2017; Yamamoto et al., 2018). Já os MRFs secundários, miogenina e MRF4 atuam como fatores de diferenciação miogênica, e mediam a expressão do fenótipo terminal (Kablar et al., 1999; Hernández-Hernández et al., 2017).

A MyoD e miogenina têm papéis fundamentais, respectivamente, nas fases precoce e tardia da miogênese durante o reparo muscular (Abreu et.al., 2017). A MyoD aparece em um estágio inicial de ativação e proliferação de mioblastos, participando ativamente até a diferenciação de tais células. Já a miogenina está envolvida em um estágio posterior na diferenciação (Hernández-Hernández et al., 2017).

Russo et al. (2010), provocaram desnervação em músculo de ratos Wistar e observaram que esse processo leva a altas taxas de degradação de proteínas e atrofia muscular e que a MyoD tem uma importante função relacionada à ativação e proliferação de células satélites na regeneração muscular.

A ativação e proliferação das CS são marcadas pela expressão aumentada da proteína MyoD, com pico em sua produção decorridos três dias após uma lesão. A indução de MyoD e Myf6 começa a ocorrer dentro de duas a seis horas da lesão e a expressão de Myf5 é mais tardia com picos dentro de 5 dias da lesão e tem expressão semelhante para miogenina (Shi \& Garry, 2006).

Assim, a atrofia muscular é decorrente do persistente estado de catabolismo muscular com proteólise do tecido e tal alteração leva a necessidade de regeneração do tecido, visto que o comprometimento do sistema muscular esquelético não só afeta a função motora, mas também influencia o comportamento emocional, a saúde mental e a qualidade de vida dos indivíduos. Desta forma, a utilização de recursos que atenuem este processo e otimizem a recuperação do tecido muscular, é de grande valia para a reabilitação. Diante deste contexto, foi levantada a hipótese de que a PBMT poderia constituir uma 
ferramenta terapêutica promissora para o tratamento de doenças que têm a atrofia muscular como consequência (Kou et al., 2019).

\section{Terapia por fotobiomodulação (PBMT)}

A PBMT tem sido considerada uma técnica segura e eficiente para o tratamento clínico de uma variedade de doenças e distúrbios musculoesqueléticos uma vez que possui propriedades analgésicas, moduladora da inflamação e reparadoras (Nakano et al., 2009).

Sabe-se que a PBMT passou por diferentes denominações na literatura científica nos últimos anos, destacando a terapia laser de baixa intensidade (LLLT), laser de baixa potência, laserterapia e fototerapia (Anders et al., 2015). De acordo com a literatura atual, existem dois principais motivos para o uso do termo PBMT: a terapia não abrange somente a luz por laser (monocromática e coerente), mas também os diodos emissores de luz (LEDs - não coerente) como esse tipo de terapia; a terapia é capaz de gerar efeitos de inibitórios a nível celular, bem como efeitos de estimulatórios, modulando processos biológicos (Heiskanen \& Hamblin, 2018). Desta forma, temos por definição que a PBMT é uma terapia atérmica realizada através de luz não ionizantes, incluindo lasers ou LEDs, no espectro visível e invisível uma (de Freitas \& Hamblin, 2016).

Foi comprovado que a interação da luz vermelha e infravermelha próxima com o tecido ocorre através de cromóforos endógenos (fotorreceptores primários), com destaque para o centros de cobre da citocromo c oxidase (CCO), IV complexo da cadeia de transporte de elétrons mitocondriais, ocasionando efeitos biofísicos e bioquímicos e consequentes efeitos terapêuticos (Anders et al., 2015; Karu, 2010).

Sabe-se que a PBMT é capaz de aumentar a produção de adenosina trifosfato (ATP), aumentar o metabolismo energético da célula, modular condições inflamatórias e o estresse oxidativo e nitrativo do tecido, assim como alterar a expressão de genes específicos (Heiskanen \& Hamblin, 2018; Hamblin, 2017, 2018).

Entretanto, deve-se destacar que o potencial terapêutico desta modalidade é dependente da aplicação de parâmetros de tratamento adequados, tais como, comprimento de onda, densidade de energia, potência, irradiância, número e tempo da sessão, assumindo a existência de uma dosimetria ótima para sua ação biomoduladora.

Desta forma, a PBMT é realizada utilizando comprimentos de onda na faixa de 600 a 700nm e 780 a $1100 \mathrm{~nm}$, pulsada ou contínua, densidade de potência (irradiância) entre 1 a $5 \mathrm{~W} / \mathrm{cm}^{2}$ e potência de saída entre $1 \mathrm{~mW}$ a $500 \mathrm{~mW}$ para evitar efeitos térmicos nos tecidos (Huang et al., 2009).

Os mecanismos da PBMT sobre o tecido muscular ainda não estão muito bem elucidados, entretanto, pode-se observar na literatura atual que o mesmo que a luz é capaz de gerar efeitos benéficos neste tecido, tais como promover melhora no metabolismo energético, ganho de massa muscular (síntese de proteínas miofibrilares), regeneração muscular, redução do dano e dor muscular na fase inicial e tardia, assim como atenuação do processo de atrofia muscular (Ferraresi et al., 2012; Ferraresi et al., 2015; Ferraresi et al., 2016).

\section{Metodologia}

Trata-se de uma revisão narrativa da literatura, de caráter descritivo exploratória, que é um tipo de estudo apropriado para descrever e discutir o desenvolvimento ou o "estado da arte" de um determinado assunto, sobre ponto de vista teórico ou contextual. Constituem, basicamente, de análise da literatura publicada de artigos de revistas impressas e/ou eletrônicas, na interpretação e análise crítica pessoal dos autores, permitindo assim, a aquisição e atualização do conhecimento sobre uma temática específica em curto espaço de tempo (Macedo et al., 2020; Moraes et al., 2020; Santiago et al., 2020).

Todos os estudos e relatórios primários que avaliaram os efeitos da PBMT na atrofia foram incluídos nesta revisão, independentemente de seus desenhos, assim como os principais estudos que descreveram os mecanismos de ação da terapia. 
Devido à novidade do projeto e aos poucos dados disponíveis sobre o assunto, não foram aplicados critérios de exclusão para evitar a falta de dados e maximizar a abrangência da pesquisa.

O processo de busca foi realizado de forma independente por dois revisores (LA. e S.S.). As bases de dados bibliográficas consultadas foram PubMed/MEDLINE, Biblioteca Virtual de Saúde (BVS) e SciELO. A busca se deu por artigos baseados em evidências publicados até 10 de dezembro de 2020 e as principais palavras-chave utilizadas para a revisão foram: Fotobiomodulação; Laser; atrofia muscular. Nenhuma limitação de idioma foi aplicada e os dados publicados em outros idiomas que não o inglês foram traduzidos usando sites e software de tradução.

\section{Resultados e Discussão}

Embora o músculo esquelético seja um tecido que alto conteúdo de miofibras multinucleadas, diversas adaptação moleculares, morfológica e funcional são necessárias para sua recuperação após um processo de atrofia muscular (Musarò \& Carosio, 2017). As células satélite, uma população de células-tronco musculares esqueléticas, são geralmente reconhecidas como a principal e, possivelmente, a única fonte de regeneração muscular pós-natal (Frontera \& Ochala, 2015). Estudos anteriores revelaram o potencial da PBMT em promover a proliferação de células satélites e inibir sua apoptose in vitro (Bendov et al., 1999; Shefer et al., 2001; Shefer et al., 2002). Ben-Dov et al., (1999) determinaram, o efeito de PBMT no comprimento de onda vermelho $(632,8 \mathrm{~nm})$ em células miogênicas de rato de cultura primária e os resultados demonstraram claramente o efeito da irradiação na indução da expressão de proteínas reguladoras do ciclo celular, aumentando assim a proliferação celular. Utilizando o mesmo comprimento de onda, Shefer at al. (2001) evidenciaram que a PBMT no comprimento de onda vermelho está envolvida na regulação de proteínas presentes na etapa inicial do processo de síntese protéica de mioblastos. Os mesmos autores observaram que a média do número de células por fibra foi triplicodo no grupo irradiado com comparação ao grupo que não recebeu irradiação, relatando um papel protetor da PBMT contra a apoptose de células satélites (Shefer et al., 2002).

Por meio de suas propriedades fotoquímicas e fotobiológicas, a aplicação da PBMT na estimulação da regeneração muscular, tem sido objeto de intenso estudo científico nos últimos anos (Amaral et al., 2001; Mesquita-Ferrari et al., 2011; Ramos et al., 2012). Nesses trabalhos, observa-se a utilização da PBMT dentro das faixas destinadas à luz visível e/ou infravermelho próximo. Dentro deste contexto, a principal atuação no tecido muscular relaciona-se ao efeito de modular o processo inflamatório e estimular a proliferação de células satélites responsáveis pela miogênses. Principalmente nos períodos iniciais pós-trauma, uma vez que a inflamação é atenuada e as células miogênicas estimuladas, há possibilidade maior de ocorrer regeneração muscular. Estudos em diversos modelos mostram que esta abordagem terapêutica é capaz de induzir a expressão de proteínas reguladoras do ciclo celular e ativar células satélites (Ben-Dov et al., 1999; Shefer et al., 2001; Nakano et al., 2009), estimular formação de miotubos (Bibikova \& Oron, 1994), promover a angiogênese (Iyomassa et al., 2009), aumentar o número e de fibras em regeneração e a densidade e atividade mitocondrial (Nakano et al., 2009; Amaral et al. 2001; Silveira et al., 2009), além de melhorar a organização de fibras musculares regeneradas (Cressoni et al., 2008).

Foi possível identificar na literatura um efeito protetor do PBMT na atrofia induzida por desnervação diversos estudos experimentais (Gigo-Benato et al., 2010; Silva-Couto et al., 2012; Rochkind \& Shainberg, 2013; Shen et al., 2013; Muniz et al., 2015; Manderlbaun-Livnat et al., 2016; Andreo et al., 2020). No estudo de Gigo-Benato et al. (2010), investigouse a influência dos lasers no comprimento de onda vermelho $(660 \mathrm{~nm})$ e infravermelho $(780 \mathrm{~nm}) \mathrm{em}$ um modelo experimental de lesão por esmagamento do nervo ciático. As análises demostraram que somente o comprimento de onda vermelho foi capaz de acelerar a recuperação funcional e prevenir a atrofia muscular. Resultados similares foram idenficados por Shen et al. (2013), utilizando a PBMT (660 nm) na prevenção da atrofia muscular. Lakyová et al. (2010), utilizando a PBMT no espectro vermelho $(670 \mathrm{~nm})$ em um modelo de atrofia muscular por isquemia e reperfusão (I/R), foi possível verificar que essa 
estimulou a neovascularização, além do efeito modulador do processo inflamatório e protetor contra a atrofia muscular e necrose do músculo após a I/R. Ainda, Rochkind \& Shainberg. (2013) observaram que a PBMT também no comprimento de onda vermelho $(632,8 \mathrm{~nm})$ foi eficaz em preservar o músculo desnervado, mantendo a atividade de creatina quinase (CK) e a quantidade receptores de acetilcolina.

Ainda, é possível observar os efeitos positivos do comprimento de onda infravermelho na atenuação da atrofia muscular nos estudos de Muniz et al. (2015) e Mandelbaum-Livnat et al. (2016). Utilizando um modelo de atrofia muscular induzida por desuso (suspensão do membro pélvico), Nakano et al. (2009) demostraram que o tratamento com PBMT ocasionou no comprimento de onda infravemermelho $(830 \mathrm{~nm})$ proporcionou um aumento no diâmetro das miofibras e no número de capilares, e ainda aumentou significativamente a proliferação de células satélite e miofibras do músculo atrofiado. Ainda, Kou et al. (2019) relataram que a PBMT atenuou a progressão da atrofia muscular induzida por desuso, devido ao fato da terapia aumentar a proliferação de células satélites e proteção à apoptose da mesma. Mais recentemente, Svobodova et al. (2019) demonstraram que a PBMT ( 808 e $905 \mathrm{~nm}$ ) resultaram em efeitos positivos na preservação da atrofia muscular induzida após lesão medular. Ademais, em modelo de atrofia muscular induzida após transecção do ligamento cruzado anterior, Assis, et al., 2015, verificou que a PBMT (808 nm) foi capaz de aumentar significativamente a área da seção muscular, diminuir na densidade da fibra muscular e a expressão de atrogina-1 e Murf-1, proteína responsáveis pela proteólise da fibra muscular.

Assim, baseado em todas as evidências demostradas, acredita-se que a PBMT tanto no comprimento de onda vermelho como no infravermelho pode ser proposta como uma recurso promissor para o tratamento da atrofia muscular oriundas de diferente doenças, justificada pelo seu mecanismo de ação e à fisiopatologia da atrofia e regeneração muscular.

\section{Considerações Finais}

A atrofia do músculo esquelético é a consequência da degradação das proteínas excedendo a síntese proteica que ocorre com o envelhecimento ou relacionada à muitas doenças, e correlaciona-se com uma redução na qualidade de vida e aumento da morbidade e mortalidade. A modulação desse processo e o impulso à regeneração são necessários para a reabilitação do paciente. Baseado nas evidências demostradas, é plausível concluir que a PBMT é uma modalidade segura, eficaz e de baixo custo, sem quaisquer efeitos colaterais que possam ocasionar efeitos adversos no paciente. Desta forma, sugere-se que a PBMT combinada com à reabilitação convencional tem o potencial no tratamento da atrofia muscular a capacidade de estimular fatores regulatórios miogênicos que promovem a ativação e proliferação de células satélites e consequente aumentar a formação de fibras musculares, assim como atenuar e apoptose de celular as vias de proteólise da fibra muscular. Mesmo considerando os resultados promissores da PBMT na atrofia muscular, destacamos a importância de novos ensaios experimentais e clínicos (controlados e randomizados) para o entendimento dos mecanismos moleculares e celulares envolvidos, e principalmente da dosimetria ideal para uma aplicação segura e efetiva em pacientes com atrofia muscular.

\section{Agradecimentos}

O presente trabalho foi realizado com apoio da Coordenação de Aperfeiçoamento de Pessoal de Nível Superior- Brasil (CAPES).

\section{Referências}

Abreu, P., Leal-Cardoso, J. H., Ceccatto, V. M., \& Hirabara, S. M. (2017). Regulation of muscle plasticity and trophism by fatty acids: A short review. Revista da Associação Médica Brasileira, 63(2), 148-155. https://doi.org/10.1590/1806-9282.63.02.148

Amaral, A. C., Parizotto, N. A., \& Salvini, T. F. (2001). Dose-dependency of low-energy HeNe laser effect in regeneration of skeletal muscle in mice. Lasers in medical science, 16(1), 44-51. https://doi.org/10.1007/p100011336 
Andraus, R., Maia, L. P., de Souza Lino, A. D., Fernandes, K., de Matos Gomes, M. V., de Jesus Guirro, R. R., \& Barbieri, C. H. (2017). LLLT actives MMP2 and increases muscle mechanical resistance after nerve sciatic rat regeneration. Lasers in medical science, 32(4), 771-778. https://doi.org/10.1007/s10103$017-2169-y$

Andreo, L., Ribeiro, B. G., Alves, A. N., Martinelli, A., Soldera, C. B., Horliana, A., Bussadori, S. K., Fernandes, K., \& Mesquita-Ferrari, R. A. (2020). Effects of Photobiomodulation with Low-level Laser Therapy on Muscle Repair Following a Peripheral Nerve Injury in Wistar Rats. Photochemistry and photobiology, 10.1111/php.13255. Advance online publication. https://doi.org/10.1111/php.13255

Andreo, L., Soldera, C. A., Ribeiro, B. G., de Matos, P., Sousa, P. B., de Alcântara Araújo Amorim, W. W., Horliana, A., Bussadori, S. K., Fernandes, K., \& Mesquita-Ferrari, R. A. (2019). Effects of Photobiomodulation on Functionality in Wistar Rats with Sciatic Nerve Injury. Photochemistry and photobiology, 95(3), 879-885. https://doi.org/10.1111/php.13048

Aoki, M. S., Miyabara, E. H., Soares, A. G., Salvini, T. F., \& Moriscot, A. S. (2006). Cyclosporin-A does not affect skeletal muscle mass during disuse and recovery. Brazilian journal of medical and biological research $=$ Revista brasileira de pesquisas medicas e biologicas, 39(2), 243-251. https://doi.org/10.1590/s0100-879x2006000200011

Alves, A. N., Fernandes, K. P., Deana, A. M., Bussadori, S. K., \& Mesquita-Ferrari, R. A. (2014). Effects of low-level laser therapy on skeletal muscle repair: a systematic review. American journal of physical medicine \& rehabilitation, 93(12), 1073-1085. https://doi.org/10.1097/PHM.0000000000000158

Assis, L., Almeida, T., Milares, L. P., dos Passos, N., Araújo, B., Bublitz, C., Veronez, S., \& Renno, A. C. (2015). Musculoskeletal Atrophy in an Experimental Model of Knee Osteoarthritis: The Effects of Exercise Training and Low-Level Laser Therapy. American journal of physical medicine \& rehabilitation, 94(8), 609-616. https://doi.org/10.1097/PHM.0000000000000219

Baehr, L. M., West, D., Marshall, A. G., Marcotte, G. R., Baar, K., \& Bodine, S. C. (2017). Muscle-specific and age-related changes in protein synthesis and protein degradation in response to hindlimb unloading in rats. Journal of applied physiology (Bethesda, Md.: 1985), 122(5), 1336-1350. https://doi.org/10.1152/japplphysiol.00703.2016

Baptista, I. L., Silvestre, J. G., Silva, W. J., Labeit, S., \& Moriscot, A. S. (2017). FoxO3a suppression and VPS34 activity are essential to anti-atrophic effects of leucine in skeletal muscle. Cell and tissue research, 369(2), 381-394. https://doi.org/10.1007/s00441-017-2614-z

Beaudart, C., Biver, E., Bruyère, O., Cooper, C., Al-Daghri, N., Reginster, J. Y., \& Rizzoli, R. (2018). Quality of life assessment in musculo-skeletal health. Aging clinical and experimental research, 30(5), 413-418. https://doi.org/10.1007/s40520-017-0794-8

Ben-Dov, N., Shefer, G., Irintchev, A., Wernig, A., Oron, U., \& Halevy, O. (1999). Low-energy laser irradiation affects satellite cell proliferation and differentiation in vitro. Biochimica et biophysica acta, 1448(3), 372-380. https://doi.org/10.1016/s0167-4889(98)00147-5

Bibikova, A., Belkin, V., \& Oron, U. (1994). Enhancement of angiogenesis in regenerating gastrocnemius muscle of the toad (Bufo viridis) by low-energy laser irradiation. Anatomy and embryology, 190(6), 597-602. https://doi.org/10.1007/BF00190110

Bloemberg, D., \& Quadrilatero, J. (2012). Rapid determination of myosin heavy chain expression in rat, mouse, and human skeletal muscle using multicolor immunofluorescence analysis. PloS one, 7(4), e35273. https://doi.org/10.1371/journal.pone.0035273

Bodine, S. C., \& Baehr, L. M. (2014). Skeletal muscle atrophy and the E3 ubiquitin ligases MuRF1 and MAFbx/atrogin-1. American journal of physiology. Endocrinology and metabolism, 307(6), E469-E484. https://doi.org/10.1152/ajpendo.00204.2014

Boonyarom, O., \& Inui, K. (2006). Atrophy and hypertrophy of skeletal muscles: structural and functional aspects. Acta physiologica (Oxford, England), 188(2), 77-89. https://doi.org/10.1111/j.1748-1716.2006.01613.x

Brito, A. A., da Silveira, E. C., Rigonato-Oliveira, N. C., Soares, S. S., Brandao-Rangel, M., Soares, C. R., Santos, T. G., Alves, C. E., Herculano, K. Z., Vieira, R. P., Lino-Dos-Santos-Franco, A., Albertini, R., Aimbire, F., \& de Oliveira, A. P. (2020). Low-level laser therapy attenuates lung inflammation and airway remodeling in a murine model of idiopathic pulmonary fibrosis: Relevance to cytokines secretion from lung structural cells. Journal of photochemistry and photobiology. B, Biology, 203, 111731. https://doi.org/10.1016/j.jphotobiol.2019.111731

Brook, M. S., Wilkinson, D. J., Phillips, B. E., Perez-Schindler, J., Philp, A., Smith, K., \& Atherton, P. J. (2016). Skeletal muscle homeostasis and plasticity in youth and ageing: impact of nutrition and exercise. Acta physiologica (Oxford, England), 216(1), 15-41. https://doi.org/10.1111/apha.12532

Chung, H., Dai, T., Sharma, S. K., Huang, Y. Y., Carroll, J. D., \& Hamblin, M. R. (2012). The nuts and bolts of low-level laser (light) therapy. Annals of biomedical engineering, 40(2), 516-533. https://doi.org/10.1007/s10439-011-0454-7

Cohen, S., Nathan, J. A., \& Goldberg, A. L. (2015). Muscle wasting in disease: molecular mechanisms and promising therapies. Nature reviews. Drug discovery, 14(1), 58-74. https://doi.org/10.1038/nrd4467

Cressoni, M. D., Dib Giusti, H. H., Casarotto, R. A., \& Anaruma, C. A. (2008). The effects of a 785-nm AlGaInP laser on the regeneration of rat anterior tibialis muscle after surgically-induced injury. Photomedicine and laser surgery, 26(5), 461-466. https://doi.org/10.1089/pho.2007.2150

da Silva, C. A., Guirro, R. R., Polacow, M. L., Cancelliero, K. M., \& Durigan, J. L. (2006). Rat hindlimb joint immobilization with acrylic resin orthoses. Brazilian journal of medical and biological research $=$ Revista brasileira de pesquisas medicas e biologicas, 39(7), 979-985. https://doi.org/10.1590/s0100-879x2006000700016

de Brito, A., Alves, A. N., Ribeiro, B. G., Barbosa, D., Magalhaes, E., Fernandes, K., Bussadori, S. K., Goulardins, J. B., \& Mesquita-Ferrari, R. A. (2018). Effect of photobiomodulation on connective tissue remodeling and regeneration of skeletal muscle in elderly rats. Lasers in medical science, 33(3), 513-521. https://doi.org/10.1007/s10103-017-2392-6

de Freitas, L. F., \& Hamblin, M. R. (2016). Proposed Mechanisms of Photobiomodulation or Low-Level Light Therapy. IEEE journal of selected topics in quantum electronics: a publication of the IEEE Lasers and Electro-optics Society, 22(3), 7000417. https://doi.org/10.1109/JSTQE.2016.2561201 
Farivar, S., Malekshahabi, T., \& Shiari, R. (2014). Biological effects of low level laser therapy. Journal of lasers in medical sciences, 5(2), 58-62.

Feng, H. Z., Chen, X., Malek, M. H., \& Jin, J. P. (2016). Slow recovery of the impaired fatigue resistance in postunloading mouse soleus muscle corresponding to decreased mitochondrial function and a compensatory increase in type I slow fibers. American journal of physiology. Cell physiology, $310(1)$, C27-C40. https://doi.org/10.1152/ajpcell.00173.2015

Ferraresi, C., Kaippert, B., Avci, P., Huang, Y. Y., de Sousa, M. V., Bagnato, V. S., Parizotto, N. A., \& Hamblin, M. R. (2015). Low-level laser (light) therapy increases mitochondrial membrane potential and ATP synthesis in C2C12 myotubes with a peak response at 3-6 h. Photochemistry and photobiology, 91(2), 411-416. https://doi.org/10.1111/php.12397

Ferraresi, C., Hamblin, M. R., \& Parizotto, N. A. (2012). Low-level laser (light) therapy (LLLT) on muscle tissue: performance, fatigue and repair benefited by the power of light. Photonics \& lasers in medicine, 1(4), 267-286. https://doi.org/10.1515/plm-2012-0032

Ferraresi, C., Huang, Y. Y., \& Hamblin, M. R. (2016). Photobiomodulation in human muscle tissue: an advantage in sports performance?. Journal of biophotonics, 9(11-12), 1273-1299. https://doi.org/10.1002/jbio.201600176

Fink, J., Kikuchi, N., \& Nakazato, K. (2018). Effects of rest intervals and training loads on metabolic stress and muscle hypertrophy. Clinical physiology and functional imaging, 38(2), 261-268. https://doi.org/10.1111/cpf.12409

Fink, J., Schoenfeld, B. J., Kikuchi, N., \& Nakazato, K. (2018). Effects of drop set resistance training on acute stress indicators and long-term muscle hypertrophy and strength. The Journal of sports medicine and physical fitness, 58(5), 597-605. https://doi.org/10.23736/S0022-4707.17.06838-4

Frontera, W. R., \& Ochala, J. (2015). Skeletal muscle: a brief review of structure and function. Calcified tissue international,96(3), 183-195. https://doi.org/10.1007/s00223-014-9915-y

Gao, Y., Arfat, Y., Wang, H., \& Goswami, N. (2018). Muscle Atrophy Induced by Mechanical Unloading: Mechanisms and Potential Countermeasures. Frontiers in physiology, 9, 235. https://doi.org/10.3389/fphys.2018.00235

Gigo-Benato, D., Russo, T. L., Tanaka, E. H., Assis, L., Salvini, T. F., \& Parizotto, N. A. (2010). Effects of 660 and 780 nm low-level laser therapy on neuromuscular recovery after crush injury in rat sciatic nerve. Lasers in surgery and medicine, 42(9), 673-682. https://doi.org/10.1002/lsm.20978

Goto, K., Sakamoto, J., Nakano, J., Kataoka, H., Honda, Y., Sasabe, R., Origuchi, T., \& Okita, M. (2017). Development and progression of immobilizationinduced skin fibrosis through overexpression of transforming growth factor- 11 and hypoxic conditions in a rat knee joint contracture model. Connective tissue research, 58(6), 586-596. https://doi.org/10.1080/03008207.2017.1284823

Gruet, M., Troosters, T., \& Verges, S. (2017). Peripheral muscle abnormalities in cystic fibrosis: Etiology, clinical implications and response to therapeutic interventions. Journal of cystic fibrosis: official journal of the European Cystic Fibrosis Society, 16(5), 538-552. https://doi.org/10.1016/j.jcf.2017.02.007

Hamblin M. R. (2017). Mechanisms and applications of the anti-inflammatory effects of photobiomodulation. AIMS biophysics, 4(3), 337-361. https://doi.org/10.3934/biophy.2017.3.337

Hamblin M. R. (2016). Photobiomodulation or low-level laser therapy. Journal of biophotonics, 9(11-12), 1122-1124. https://doi.org/10.1002/jbio.201670113

Heiskanen, V., \& Hamblin, M. R. (2018). Correction: Photobiomodulation: lasers vs. light emitting diodes?. Photochemical \& photobiological sciences: Official journal of the European Photochemistry Association and the European Society for Photobiology, 18(1), 259. Advance online publication. https://doi.org/10.1039/c8pp90049c

Hernández-Hernández, J. M., García-González, E. G., Brun, C. E., \& Rudnicki, M. A. (2017). The myogenic regulatory factors, determinants of muscle development, cell identity and regeneration. Seminars in cell \& developmental biology, 72, 10-18. https://doi.org/10.1016/j.semcdb.2017.11.010

Hindi, S. M., Tajrishi, M. M., \& Kumar, A. (2013). Signaling mechanisms in mammalian myoblast fusion. Science signaling, 6(272). https://doi.org/10.1126/scisignal.2003832

Huang, Y. Y., Chen, A. C., Carroll, J. D., \& Hamblin, M. R. (2009). Biphasic dose response in low level light therapy. Dose-response : a publication of International Hormesis Society, 7(4), 358-383. https://doi.org/10.2203/dose-response.09-027.

Iyomasa, D. M., Garavelo, I., Iyomasa, M. M., Watanabe, I. S., \& Issa, J. P. (2009). Ultrastructural analysis of the low level laser therapy effects on the lesioned anterior tibial muscle in the gerbil. Micron (Oxford, England: 1993), 40(4), 413-418. https://doi.org/10.1016/j.micron.2009.02.002

Järvinen, T. A., Józsa, L., Kannus, P., Järvinen, T. L., \& Järvinen, M. (2002). Organization and distribution of intramuscular connective tissue in normal and immobilized skeletal muscles. An immunohistochemical, polarization and scanning electron microscopic study. Journal of muscle research and cell motility, 23(3), 245-254. https://doi.org/10.1023/a:1020904518336

Joglekar, S., Nau, P. N., \& Mezhir, J. J. (2015). The impact of sarcopenia on survival and complications in surgical oncology: A review of the current literature. Journal of surgical oncology, 112(5), 503-509. https://doi.org/10.1002/jso.24025

Jagoe, R. T., \& Goldberg, A. L. (2001). What do we really know about the ubiquitin-proteasome pathway in muscle atrophy?. Current opinion in clinical nutrition and metabolic care, 4(3), 183-190. https://doi.org/10.1097/00075197-200105000-00003

Kablar, B., Krastel, K., Ying, C., Tapscott, S. J., Goldhamer, D. J., \& Rudnicki, M. A. (1999). Myogenic determination occurs independently in somites and limb buds. Developmental biology, 206(2), 219-231. https://doi.org/10.1006/dbio.1998.9126

Karu T. I. (2010). Multiple roles of cytochrome c oxidase in mammalian cells under action of red and IR-A radiation. IUBMB life, 62(8), 607-610. https://doi.org/10.1002/iub.359

Kou, Y. T., Liu, H. T., Hou, C. Y., Lin, C. Y., Tsai, C. M., \& Chang, H. (2019). A transient protective effect of low-level laser irradiation against disuseinduced atrophy of rats. Lasers in medical science, 34(9), 1829-1839. https://doi.org/10.1007/s10103-019-02778-5 
Lakyová, L., Toporcer, T., Tomečková, V., Sabo, J., \& Radoňak, J. (2010). Low-level laser therapy for protection against skeletal muscle damage after ischemia-reperfusion injury in rat hind limbs. Lasers in surgery and medicine, 42(9), 665-672. https://doi.org/10.1002/lsm.20967

Lee, H. K., Rocnik, E., Fu, Q., Kwon, B., Zeng, L., Walsh, K., \& Querfurth, H. (2012). Foxo/atrogin induction in human and experimental myositis. Neurobiology of disease, 46(2), 463-475. https://doi.org/10.1016/j.nbd.2012.02.011

Lee, J. H., \& Jun, H. S. (2019). Role of Myokines in Regulating Skeletal Muscle Mass and Function. Frontiers in physiology, 10, 42. https://doi.org/10.3389/fphys.2019.00042

Macedo, D. B., Tim R. C., Macedo, J. B. S. C., Macedo, G. M., Martignago, C. C. S., \& Assis, L. (2020). Therapeutic perspective of light for coronavirus treatment. Research, Society and Development, 9(8), e766986320. http://dx.doi.org/10.33448/rsd-v9i8.6320

Mandelbaum-Livnat, M. M., Almog, M., Nissan, M., Loeb, E., Shapira, Y., \& Rochkind, S. (2016). Photobiomodulation Triple Treatment in Peripheral Nerve Injury: Nerve and Muscle Response. Photomedicine and laser surgery, 34(12), 638-645. https://doi.org/10.1089/pho.2016.4095

March, L., Smith, E. U., Hoy, D. G., Cross, M. J., Sanchez-Riera, L., Blyth, F., Buchbinder, R., Vos, T., \& Woolf, A. D. (2014). Burden of disability due to musculoskeletal (MSK) disorders. Best practice \& research. Clinical rheumatology, 28(3), 353-366. https://doi.org/10.1016/j.berh.2014.08.002

Mesquita-Ferrari, R. A., Martins, M. D., Silva, J. A., Jr, da Silva, T. D., Piovesan, R. F., Pavesi, V. C., Bussadori, S. K., \& Fernandes, K. P. (2011). Effects of low-level laser therapy on expression of TNF- $\alpha$ and TGF- $\beta$ in skeletal muscle during the repair process. Lasers in medical science, 26(3), 335-340. https://doi.org/10.1007/s10103-010-0850-5

Monte Alegre, D. C., Almeida, J. F. S., Oliveira, T. V. C., Cândido, E. A. F. (2012). Plasticidade muscular: do músculo sadio ao espastico. Fisioterapia, 2, (1), 16-34. https://doi.org/10.6008/ESS2236-9600.2012.001.0003

Moraes, J. P., Tim R. C., \& Assis, L. (2020). Considerations about the use of Ozone therapy $\left(\mathrm{O}_{3}\right)$ in the treatment of Endometriosis. Research, Society and Development, 9(9), e403997616. http://dx.doi.org/10.33448/rsd-v9i9.7616

Morley, J. E., Kalantar-Zadeh, K., \& Anker, S. D. (2020). COVID-19: a major cause of cachexia and sarcopenia?. Journal of cachexia, sarcopenia and muscle, 11(4), 863-865. https://doi.org/10.1002/jcsm.12589

Muniz, K. L., Dias, F. J., Coutinho-Netto, J., Calzzani, R. A., Iyomasa, M. M., Sousa, L. G., Santos, T. T., Teles, V., Watanabe, I. S., Fazan, V. P., \& Issa, J. P. (2015). Properties of the tibialis anterior muscle after treatment with laser therapy and natural latex protein following sciatic nerve crush. Muscle \& nerve, 52(5), 869-875. https://doi.org/10.1002/mus.24602

Musarò, A., \& Carosio, S. (2017). Isolation and Culture of Satellite Cells from Mouse Skeletal Muscle. Methods in molecular biology (Clifton, N.J.), 1553, $155-167$

Nakano, J., Kataoka, H., Sakamoto, J., Origuchi, T., Okita, M., \& Yoshimura, T. (2009). Low-level laser irradiation promotes the recovery of atrophied gastrocnemius skeletal muscle in rats. Experimental physiology, 94(9), 1005-1015. https://doi.org/10.1113/expphysiol.2009.047738

Ono S. (2010). Dynamic regulation of sarcomeric actin filaments in striated muscle. Cytoskeleton (Hoboken, N.J.),67(11), 677-692. https://doi.org/10.1002/cm.20476

Ramos, L., Leal Junior, E. C., Pallotta, R. C., Frigo, L., Marcos, R. L., de Carvalho, M. H., Bjordal, J. M., \& Lopes-Martins, R. Á. (2012). Infrared (810 nm) low-level laser therapy in experimental model of strain-induced skeletal muscle injury in rats: effects on functional outcomes. Photochemistry and photobiology, 88(1), 154-160. https://doi.org/10.1111/j.1751-1097.2011.01030.x

Rochkind, S., \& Shainberg, A. (2013). Protective effect of laser phototherapy on acetylcholine receptors and creatine kinase activity in denervated muscle. Photomedicine and laser surgery, 31(10), 499-504. https://doi.org/10.1089/pho.2013.3537

Rom, O., \& Reznick, A. Z. (2016). The role of E3 ubiquitin-ligases MuRF-1 and MAFbx in loss of skeletal muscle mass. Free radical biology \& medicine, 98, 218-230. https://doi.org/10.1016/j.freeradbiomed.2015.12.031

Rosa-Caldwell, M. E., \& Greene, N. P. (2019). Muscle metabolism and atrophy: let's talk about sex. Biology of sex differences, 10(1), 43. https://doi.org/10.1186/s13293-019-0257-3

Russo, T. L., Peviani, S. M., Durigan, J. L., Gigo-Benato, D., Delfino, G. B., \& Salvini, T. F. (2010). Stretching and electrical stimulation reduce the accumulation of MyoD, myostatin and atrogin-1 in denervated rat skeletal muscle. Journal of muscle research and cell motility, 31(1), 45-57. https://doi.org/10.1007/s10974-010-9203-z

Santiago, E. J. P., Freire, A. K. S., Ferreira, D. S. A., Amorim, J. F., Cunha, A. L. X., Freitas, J. R., Silva, A. S. A., Moreira, G. R., Cantalice, J. R. B., \& Cunha Filho, M. (2020). Velocity of deaths and confirmed cases of COVID-19 in Brazil, Italy and worldwide. Research, Society and Development, 9(7), e263974085. https://doi.org/10.33448/rsd-v9i7.4085

Schiaffino, S., Dyar, K. A., Ciciliot, S., Blaauw, B., \& Sandri, M. (2013). Mechanisms regulating skeletal muscle growth and atrophy. The FEBS journal, 280(17), 4294-4314. https://doi.org/10.1111/febs. 12253

Schiaffino, S., \& Reggiani, C. (2011). Fiber types in mammalian skeletal muscles. Physiological reviews, 91(4), 1447-1531. https://doi.org/10.1152/physrev.00031.2010

Scicchitano, B. M., Faraldi, M., \& Musarò, A. (2015). The Proteolytic Systems of Muscle Wasting. Recent advances in DNA \& gene sequences, 9(1), 26-35. https://doi.org/10.2174/2352092209999150911121502 
Silva-Couto, M. A., Gigo-Benato, D., Tim, C. R., Parizotto, N. A., Salvini, T. F., \& Russo, T. L. (2012). Effects of low-level laser therapy after nerve reconstruction in rat denervated soleus muscle adaptation. Revista brasileira de fisioterapia (Sao Carlos (Sao Paulo, Brazil)), 16(4), 320-327. https://doi.org/10.1590/s1413-35552012005000035

Silveira, P. C., Silva, L. A., Fraga, D. B., Freitas, T. P., Streck, E. L., \& Pinho, R. (2009). Evaluation of mitochondrial respiratory chain activity in muscle healing by low-level laser therapy. Journal of photochemistry and photobiology. B, Biology, 95(2), 89-92. https://doi.org/10.1016/j.jphotobiol.2009.01.004

Shefer, G., Oron, U., Irintchev, A., Wernig, A., \& Halevy, O. (2001). Skeletal muscle cell activation by low-energy laser irradiation: a role for the MAPK/ERK pathway. Journal of cellular physiology, 187(1), 73-80. https://doi.org/10.1002/1097-4652(2001)9999:9999<::AID-JCP1053>3.0.CO;2-9

Shefer, G., Partridge, T. A., Heslop, L., Gross, J. G., Oron, U., \& Halevy, O. (2002). Low-energy laser irradiation promotes the survival and cell cycle entry of skeletal muscle satellite cells. Journal of cell science, 115(Pt 7), 1461-1469.

Shen, C. C., Yang, Y. C., \& Liu, B. S. (2013). Effects of large-area irradiated laser phototherapy on peripheral nerve regeneration across a large gap in a biomaterial conduit. Journal of biomedical materials research. Part A, 101(1), 239-252. https://doi.org/10.1002/jbm.a.34314

Shi, X., \& Garry, D. J. (2006). Muscle stem cells in development, regeneration, and disease. Genes \& development, 20(13), 1692-1708. https://doi.org/10.1101/gad.1419406

Spangenburg, E. E., \& Booth, F. W. (2003). Molecular regulation of individual skeletal muscle fibre types. Acta physiologica Scandinavica, 178(4), 413-424. https://doi.org/10.1046/j.1365-201X.2003.01158.x

Svobodova, B., Kloudova, A., Ruzicka, J., Kajtmanova, L., Navratil, L., Sedlacek, R., Suchy, T., Jhanwar-Uniyal, M., Jendelova, P., \& Machova Urdzikova, L. (2019). The effect of $808 \mathrm{~nm}$ and $905 \mathrm{~nm}$ wavelength light on recovery after spinal cord injury. Scientific reports, 9(1), 7660 https://doi.org/10.1038/s41598-019-44141-2

Terena, S., Fernandes, K., Bussadori, S. K., Brugnera Junior, A., de Fátima Teixeira da Silva, D., Magalhães, E., \& Ferrari, R. (2018). Infrared Laser Improves Collagen Organization in Muscle and Tendon Tissue During the Process of Compensatory Overload. Photomedicine and laser surgery, 36(3), 130-136. https://doi.org/10.1089/pho.2017.4302

Wall, B. T., Dirks, M. L., Snijders, T., Senden, J. M., Dolmans, J., \& van Loon, L. J. (2014). Substantial skeletal muscle loss occurs during only 5 days of disuse. Acta physiologica (Oxford, England), 210(3), 600-611. https://doi.org/10.1111/apha.12190

Wall, B. T., \& van Loon, L. J. (2013). Nutritional strategies to attenuate muscle disuse atrophy. Nutrition reviews, 71(4), 195-208. https://doi.org/10.1111/nure.12019

Wang, Y., Zhou, Y., \& Graves, D. T. (2014). FOXO transcription factors: their clinical significance and regulation. BioMed research international, 2014, 925350. https://doi.org/10.1155/2014/92535

Wang, J., Wang, F., Zhang, P., Liu, H., He, J., Zhang, C., Fan, M., \& Chen, X. (2017). PGC-1 $\alpha$ over-expression suppresses the skeletal muscle atrophy and myofiber-type composition during hindlimb unloading. Bioscience, biotechnology, and biochemistry,81(3), 500-513. https://doi.org/10.1080/09168451.2016.1254531

Zammit P. S. (2017). Function of the myogenic regulatory factors Myf5, MyoD, Myogenin and MRF4 in skeletal muscle, satellite cells and regenerative myogenesis. Seminars in cell \& developmental biology, 72, 19-32. https://doi.org/10.1016/j.semcdb.2017.11.011

Zhang, P., Chen, X., \& Fan, M. (2007). Signaling mechanisms involved in disuse muscle atrophy. Medical hypotheses, 69(2), 310-321. https://doi.org/10.1016/j.mehy.2006.11.043

Yamamoto, M., Legendre, N. P., Biswas, A. A., Lawton, A., Yamamoto, S., Tajbakhsh, S., Kardon, G., \& Goldhamer, D. J. (2018). Loss of MyoD and Myf5 in Skeletal Muscle Stem Cells Results in Altered Myogenic Programming and Failed Regeneration. Stem cell reports, 10(3), 956-969. https://doi.org/10.1016/j.stemcr.2018.01.027

Yoshiko, A., Yamauchi, K., Kato, T., Ishida, K., Koike, T., Oshida, Y., \& Akima, H. (2018). Effects of post-fracture non-weight-bearing immobilization on muscle atrophy, intramuscular and intermuscular adipose tissues in the thigh and calf. Skeletal radiology, 47(11), 1541-1549. https://doi.org/10.1007/s00256018-2985-6

You, J. S., Anderson, G. B., Dooley, M. S., \& Hornberger, T. A. (2015). The role of mTOR signaling in the regulation of protein synthesis and muscle mass during immobilization in mice. Disease models \& mechanisms, 8(9), 1059-1069. https://doi.org/10.1242/dmm.019414 